\title{
Sensación de confort térmico en ambientes educativos en la zona altoandina de Puno
}

Sensation of thermal comfort in school environments in the high-andean zone of Puno

Recibido: marzo 31 de 2021 | Revisado: junio 15 de 2021 | Aceptado: agosto 20 de 2021

Hugo A. Ccama Condori ${ }^{\mathrm{I}}$

1 Universidad Nacional del Altiplano, Facultad de Ingeniería Civil y Arquitectura, Puno-Perú

Arquitecto, Ingeniero Civil, Doctor en Ciencia Tecnología y Medio Ambiente

Autor para correspondencia E-mail: hccama@unap.edu.pe

\section{RESUMEN}

Se estimó la sensación térmica percibida en relación al confort térmico en ambientes escolares de la zona altoandina de Puno. El estudio se realizó bajo el enfoque adaptativo del confort, se aplicó encuestas a estudiantes de acuerdo con la escala de sensaciones térmicas percibidas, con el uso en paralelo de un termómetro que registra la temperatura ambiente; el registro de datos se realizó en un aula convencional, así como en un aula modificada, posterior al levantamiento de datos, se realizó el análisis estadístico de medias por intervalo de sensación térmica y se aplicó la regresión lineal de los datos registrados, para obtener el valor neutro y rango de confort térmico para el periodo más crítico. Los resultados revelaron una temperatura neutral media de $8,6^{\circ} \mathrm{C}$ para el aula convencional y $12,82^{\circ} \mathrm{C}$ para el aula modificada. Esto evidencia que el sujeto de estudio considera como temperatura neutral a la situación de adaptación del individuo en un ambiente determinado, así mismo los cálculos de línea de regresión media evidenciaron que existe una relación muy alta entre la sensación térmica percibida y temperatura del ambiente con valores que representan 0,9264 para el aula convencional y 0,9144 para el aula modificada, por lo que se concluye que el confort térmico está directamente relacionado con la sensación de bienestar del individuo en un ambiente.

Palabras clave: ambiente térmico; confort térmico; sensación térmica

\begin{abstract}
The perceived thermal sensation in relation to thermal comfort in school environments in the high Andean zone of Puno was estimated. The adaptive approach to thermal comfort was assumed, for which surveys were applied according to the scale of perceived sensations, with the simultaneous use of a thermometer that records ambient temperature data; The data recording was carried out in a conventional classroom, as well as in a modified classroom, after the data collection, the statistical analysis of means by thermal sensation interval was carried out and the linear regression of the registered data
\end{abstract}

\footnotetext{
(C) Los autores. Este artículo es publicado por la Revista Campus de la Facultad de Ingeniería y Arquitectura de la Universidad de San Martín de Porres. Este artículo se distribuye en los términos de la Licencia Creative Commons Atribución No-comercial - Compartir-Igual 4.0 Internacional (https://creativecommons.org/licenses/ CC-BY), que permite el uso no comercial, distribución y reproducción en cualquier medio siempre que la obra original sea debidamente citada. Para uso comercial contactar a: revistacampus@usmp.pe.
}

https: 
was applied, to obtain the neutral value and thermal comfort range for the most critical period. The results revealed that the mean neutral temperature for the conventional classroom was $8.6^{\circ} \mathrm{C}$ while for the modified classroom it presented a calculated value of $12.82^{\circ} \mathrm{C}$, these values reveal that the study subject considers the condition of adaptation of the individual in a given environment, likewise the mean regression line calculations showed that there is a very high relationship between the thermal sensation and the temperature of the environment with values that represent 0.9264 for the conventional classroom and 0.9144 for the modified classroom, Therefore, it is concluded that thermal comfort is directly related to the feeling of wellbeing of the individual in a thermal environment.

Key words: Thermal environment; thermal comfort; thermal sensation

\section{Introducción}

El manejo del confort térmico en ambientes escolares en zonas altoandinas, a nivel nacional e internacional se ha convertido, en la actualidad, en uno de los problemas más importantes para su estudio debido a las condiciones climáticas extremas que vive la población. Los ambientes escolares son espacios donde los estudiantes pasan gran parte de su tiempo, lo que implica que estas deben ofrecer las condiciones apropiadas de confort térmico. Sin embargo, en las zonas alto andinas del Perú, los ambientes escolares no ofrecen las condiciones térmicas adecuadas para el correcto desempeño de actividades educativas y debido a estas condiciones adversas de las aulas y la prolongada exposición a bajas temperaturas durante los horarios de clase disminuye la capacidad de aprendizaje y productividad de los estudiantes lo que afecta a su desarrollo educativo.

Por otro lado, es pertinente mencionar que gran número de investigaciones abordan la problemática del desarrollo educativo desde el enfoque teórico pedagógico como si fuera el único aspecto que influye sobre el desarrollo educativo, no dando importancia a las condiciones de confort térmico de los ambientes escolares que están directamente relacionados con el desarrollo educativo; esta es la razón por la cual este estudio aborda dicha problemática del confort térmico, cuyos resultados permitirán implementar estrategias de mejoras en estos ambientes escolares a partir del conocimiento de la sensación térmica percibida por los estudiantes en estos ambientes.

En el ámbito internacional se han realizado estudios similares con valoraciones de sensación térmica y medición simultánea de temperatura en espacios exteriores, mas no en espacios interiores; sin embargo, se ha encontrado en el contexto latinoamericano estudios de evaluación térmica en aulas escolares en zonas de altura basados solo en mediciones de temperatura de confort, entre las que destacan son los estudios de Basso et al., 2000; Mercat, et al. 2016; Walter et al., 2011. En el ámbito local no existen estudios similares. En ese sentido, este trabajo se convierte en un estudio inédito que contribuye al abordaje de esta temática de sensación de confort térmico en ambientes escolares. 
Los ambientes escolares educativos ofrecen una decisiva influencia sobre los procesos de enseñanza y aprendizaje de estudiantes y docentes, Shamsuddin, 2012. Así también, se señala que un mal ambiente escolar interfiere en el rendimiento y productividad y puede causar disconfort e irritación, así como problemas de salud en escolares, profesores y personal (Del Campo y Mendivil, 2003). Así mismo, numerosos estudios realizados han demostrado que el rendimiento del individuo se ve afectado negativamente por el disconfort del ambiente (Wargocki y Wyon, 2006; Maxwell, 2016; Pellegrino, 2015; Ledesma, et.al. 2015). Lo que permite afirmar que el ambiente térmico influye, decididamente, en el desarrollo educativo de los estudiantes.

El confort térmico, desde el punto de vista fisiológico, es el balance de energía entre el cuerpo humano y su ambiente térmico (Fanger, 1973). Así mismo, desde el punto de vista psicológico es la sensación del sujeto, entendiéndose como la "condición de la mente en la que se expresa la satisfacción con un ambiente térmico" (ISO 7730, 2005), No obstante, una definición apropiada sería aquella que contiene tanto el factor fisiológico como psicológico, de acuerdo a Bojórquez (2010) El confort térmico está fundamentado en las sensaciones fisiológicas y psicológicas generadas por los estímulos del ambiente térmico. Así mismo, se expresa como "la satisfacción psicofisiológica del ser humano con respecto al ambiente térmico" (Nikolopoulou y Steemers, 2003).

En ese sentido, el confort térmico está directamente relacionado con la sensación de bienestar fisiológico y psicológico del individuo en un ambiente térmico, aspecto fundamental que se debe tener en cuenta al momento de proyectar una edificación, una prioridad importante es que ésta genere condiciones térmicas confortables y que los usuarios, en términos generales, no sientan ni frío ni calor (Miranda, 2008).

Para el estudio del confort térmico existen dos enfoques teóricos: a) enfoque predictivo y b) enfoque adaptativo (Nicol y Humphreys, 1998). En el enfoque predictivo, la investigación sobre confort se realiza en condiciones de pruebas controladas, básicamente, en cámaras meteorológicas; el individuo es aislado y se manipula su condición psicológica y funcionamiento fisiológico (Bojórquez, 2010). Por otro lado, el enfoque adaptativo es un índice de confort térmico que parte del estudio del individuo en su hábitat natural por lo que no se puede manipular su aspecto psicológico ni fisiológico (Rincón y Bojórquez, 2014).

Humphreys y Nicol (1998) propusieron el enfoque adaptativo donde fundamentalmente se establece la temperatura de neutralidad como temperatura de confort en ambientes interiores. Este estudio asume el enfoque adaptativo como fundamento teórico.

La estimación del confort térmico en el enfoque adaptativo está relacionada con la sensación térmica percibida. Estas percepciones están en función de diferentes aspectos propuestos en modelos biofísicos basados en leyes termodinámicos (Tsuzuki y Ohfuku, 2002). Desde el punto de vista fisiológico, el confort térmico es el estado de equilibrio indicado por el balance térmico del cuerpo humano, entendiéndose como la pérdida o ganancia de energía originada 
por el metabolismo y la termorregulación (Fanger, 1973). Así mismo, la sensación de confort térmico es percibida por el usuario según su grado de adaptación en el espacio donde se encuentra realizando sus actividades (Móndelo et al., 2001).

Por otro lado, la adaptación térmica se puede considerar como "el decremento gradual de respuesta del organismo a repetidas exposiciones a estímulos que se reciben de un medio ambiente específico" (Nikolopoulou y Steemers, 2003).

En ese contexto, el confort térmico es uno de los aspectos importantes que se tiene que considerar en el diseńo de la envolvente de los ambientes escolares. Lograr el bienestar fisiológico y psicológico es el objetivo primordial a la hora de diseñar y construir cualquier ambiente como hábitat del ser humano. En ese propósito, este estudio buscó estimar el confort térmico en dos ambientes escolares denominados: aula convencional y aula modificada con tecnologías bioclimáticas que permitirá en el futuro mejorar las estrategias de diseño a fin de que estas presenten adecuadas condiciones de confort. En ese sentido, el objetivo planteado fue estimar la sensación térmica percibida en relación al confort térmico en una aula convencional y en otra modificada con tecnologías bioclimáticas en la zona altoandina de Puno.

\section{Métodos}

El ambiente escolar objeto de estudio pertenece a la Institución Educativa Secundaria Pacaje (I.E.S. Pacaje). Esta se encuentra ubicada en la zona altoandina del Perú, específicamente en la localidad de Pacaje ( $14^{\circ} 04^{\prime} 12^{\prime \prime}$ latitud sur, $70^{\circ}$ 26' 20" longitud oeste a una altitud de 4345 m.s.n.m.). Pertenece al distrito de Macusani, provincia Carabaya, región Puno. El lugar corresponde a la unidad geográfica de la puna de acuerdo a la clasificación de regiones naturales (Pulgar, 1987). Así mismo, la zona de estudio tiene un clima frígido y glacial, con temperaturas muy bajas inferiores a $0^{\circ} \mathrm{C}$. En la Figura 1 se muestra la zona de estudio.

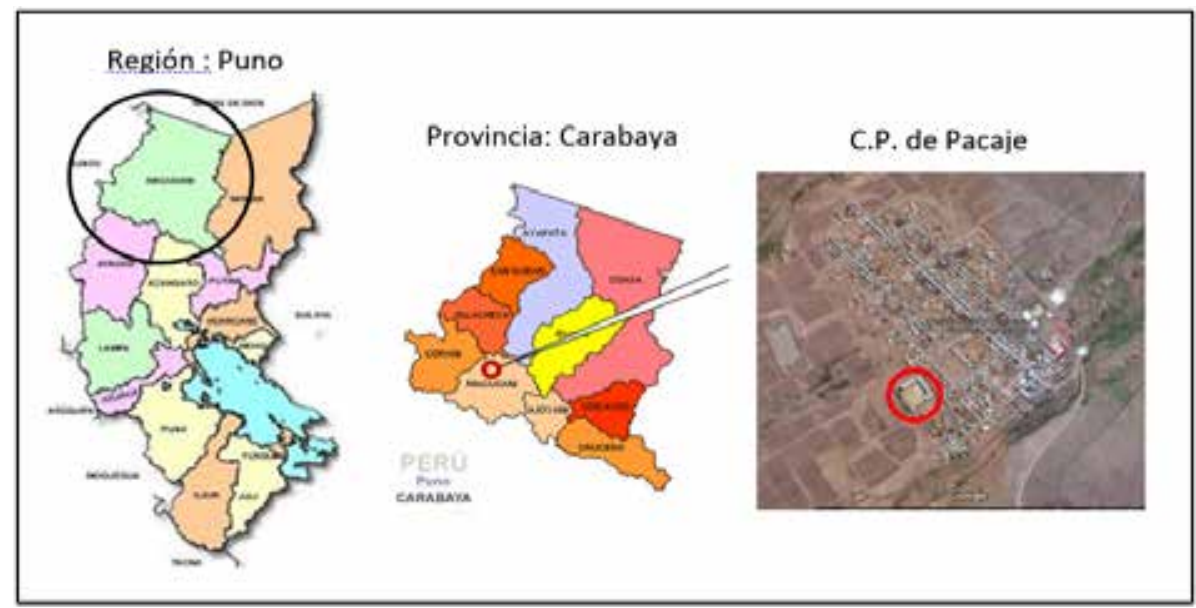

Figura 1. Localización de la zona de estudio, I.E.S. Pacaje

El aula convencional tuvo como envolvente muros $y$ tabiques de albañilería, tarrajeo de cemento-arena con un espesor de $2.00 \mathrm{~cm}$. tanto en la parte exterior como en la parte interior. La cobertura fue de calamina galvanizada 
sujeta en estructuras de tijerales de madera. El cielorraso, de material triplay de $4 \mathrm{~mm}$. Los pisos, de madera machihembrado; las ventanas de vidrio simple incoloro transparente de 2" y la puerta de madera.

El aula modificada tuvo como envolvente muros de albañilería tratados con tecnopor de 2" (poliestireno expandido) revestidos con placas de yeso. El cielo raso fue tratado con poliestireno expandido de 2", acabado final de placas de superboard. El piso, tratado con tecnopor de 2" con acabado final de madera machihembrada y las ventanas, tratadas con vidrios dobles. Así mismo, se incluyeron paneles solares que funcionan con colector solar.

Se asumió el enfoque adaptativo de confort térmico para lo cual se aplicaron encuestas a 50 estudiantes de acuerdo a la escala de sensaciones térmicas percibidas según la norma ISO 10551, 1995 con el uso simultáneo de un termómetro que registra datos de temperatura ambiente.

El registro de datos se realizó en el aula convencional; así como en el aula modificada. Después del levantamiento de datos, se realizó el análisis estadístico de medias por intervalo de sensación térmica y aplicó la regresión lineal de los datos registrados para obtener el valor neutro y rango de confort térmico para el periodo más crítico (mes de junio).

El análisis de correlación de datos entre la sensación térmica percibida y la temperatura de ambiente se realizó con el método de medias por intervalo de sensación térmica (MIST), recomendada por (Gómez-Azpeitia et al., 2007). Esta metodología hace uso de la estadística descriptiva en el cálculo del valor neutral de temperatura que es considerada como confort térmico del ambiente. La representación esquemática del método mencionado se presenta en la Figura 2.

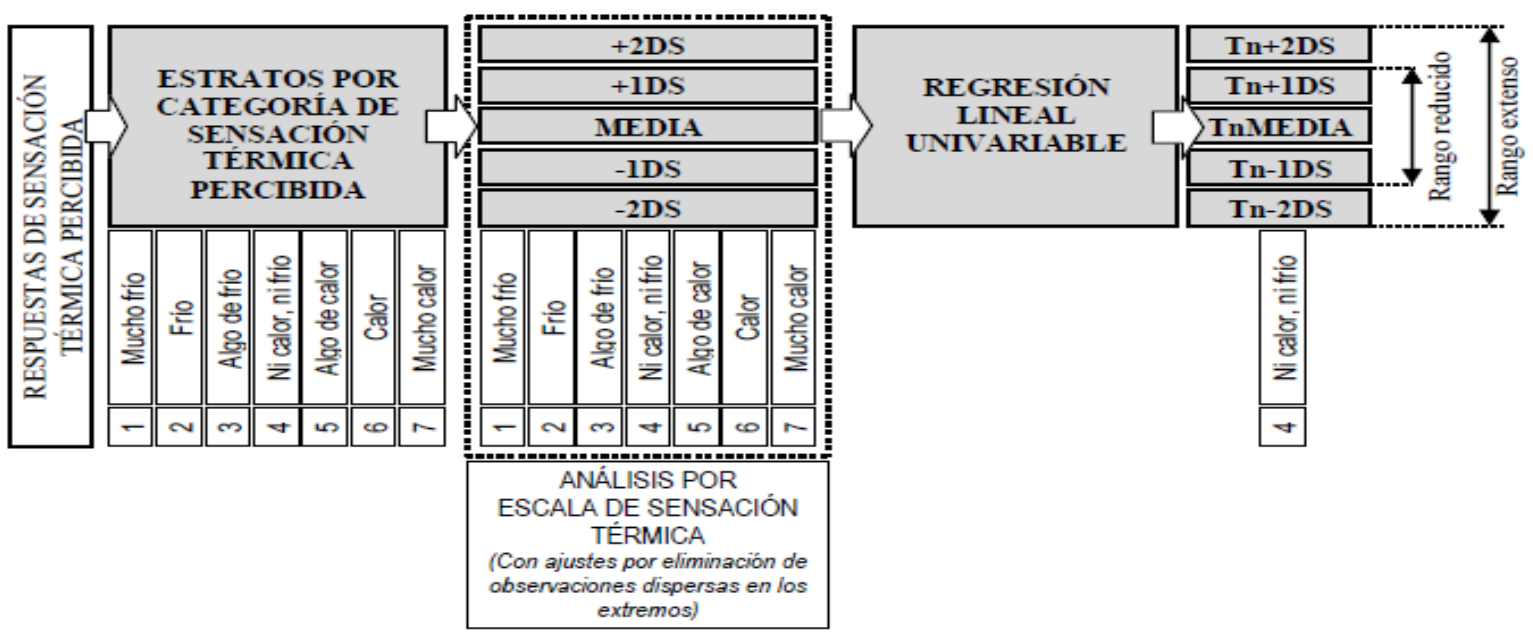

Figura 2. Estimación de temperatura neutral (Tn) con el método de medias por intervalo de sensación térmica percibida

Nota. Gómez-Azpeitia et al., 2007

Con el objetivo de obtener la línea de regresión se establecieron grupos de datos para calcular el valor promedio y la desviación estándar. De este modo, la regresión lineal no se realiza con la totalidad de los pares de registros de 
la muestra, sino se ejecuta con valores medios y los rangos se determinan mediante la suma y resta de una o más veces de la desviación estándar (DS) de la muestra (Brager y De Dear, 1998). Así mismo, se ha efectuado el procesamiento Tabla 1

Escala de sensación térmica percibida, 1995 por separado de los datos recolectados de acuerdo a las categorías de respuesta de confort según la norma ISO 10551. Para ello, se determinó los valores promedio y desviación estándar de las temperaturas registradas para cada respuesta (Tabla 1).

\begin{tabular}{cl}
\hline Escala & Sensación térmica terminal \\
\hline 7 & Mucho calor \\
6 & Calor \\
5 & Algo de calor \\
4 & Ni calor, ni frío \\
3 & Algo de frío \\
2 & Frío \\
1 & Mucho frío \\
\hline
\end{tabular}

Nota. ISO 10551, 1995

Después de obtener estos datos se establecieron los rangos de distribución para cada categoría de respuesta. Esta se realizó en función de la temperatura media y la adición \pm 1 DS, \pm 2 DS. Una vez obtenido estos valores se realizó la regresión lineal, correspondiente a la temperatura neutral media. De la misma forma se determinaron las rectas de los límites $\pm 2 \mathrm{DS}$, y a los límites $\pm 1 \mathrm{DS}$ y por último se obtuvieron los coeficientes de determinación para los valores medios, \pm 2 DS y $\pm 1 \mathrm{DS}$, para efectos de validez del modelo se consideró el valor de la media. También se calcularon valores para $\mathrm{R}^{2}$. Los cálculos se realizaron en el programa Excel. Para la interpretación de los cálculos logrados en función a las encuestas y registro de temperatura se consideró pertinente analizar dos aspectos que se detallan a continuación:

El efecto por variación de magnitud de la temperatura: para evaluar este aspecto se estableció como valor de referencia la pendiente de la recta $(m)$, y se planteó lo siguiente:

a) Entre más se aleje el valor de $\mathrm{m}$ del cero, más influencia tiene la variable $\mathrm{X}$, sobre la $\mathrm{Y}$.

b) Si $\mathrm{m}=0$ entonces $Y$ es constante.

c) $\mathrm{Sim}=\infty$ entonces $X$ es una constante que no afecta el valor de $Y$.

d) Si el valor de $\mathrm{m}$ es positivo, la relación de variables es directamente proporcional, entonces se interpreta que entre más aumente la variable $\mathrm{X}$, más aumenta también la variable Y, o sea hay un aumento hacia la sensación térmica de calor.

e) Si el valor de $\mathrm{m}$ es negativo, la relación de variables es inversamente proporcional, entonces se puede interpretar que entre más aumente la variable $\mathrm{X}$, la variable $\mathrm{Y}$ más reduce su valor, o sea hay un aumento hacia la sensación térmica de frío.

La claridad de la percepción por los sujetos de estudio: en este aspecto fue importante establecer la homogeneidad de la muestra. Se tomó como referencia 
el coeficiente de determinación $\mathrm{R}^{2}$ que sirvió para conocer el grado de dispersión de la respuesta con base en los estudios de Humphreys et al., 2007; Nikolopoulou, 2004 y Ruiz, 2007. Se estableció los parámetros de los rangos de las condiciones:

a) Si la $\mathrm{R}^{2} \geq 0.9$ la correlación es muy alta ya que hay certeza en la concentración de respuestas. La muestra no es dispersa.

b) Si $0.7 \leq \mathrm{R}^{2}<0.9$ se puede decir que la correlación es alta, la muestra es poco dispersa.

c) Si $0.5 \leq \mathrm{R}^{2}<0.7$, la correlación es media, la muestra tiene una concentración media.

d) Si la $\mathrm{R}^{2}<0.5$, la correlación es baja con alto grado de dispersión en la muestra indica que no hay claridad en la percepción de la sensación térmica. En caso de que un valor de $m$ presente una correlación baja, se considerará más apropiado el próximo inmediato con un valor de $\mathrm{R}^{2}$ de al menos una correlación media $\left(0.5 \leq \mathrm{R}^{2}<0.7\right)$.

\section{Resultados y Discusión}

La temperatura neutral media para el aula convencional fue de $8,6^{\circ} \mathrm{C}$ mientras que para el aula modificada, $12,82^{\circ} \mathrm{C}$. Estos valores revelan que los estudiantes sujetos de estudio consideran como temperatura neutral a la condición de adaptación en un determinado ambiente por lo que se afirma que la sensación térmica está relacionada con la adaptación térmica que tiene el sujeto con su medio ambiente.

Esto coincide con el estudio realizado por Nikolopouloua y Steemers, 2003. Por otro lado, la línea de regresión media evidenció que existe una relación muy alta y positiva entre la sensación térmica y temperatura del ambiente con valores de 0,9264 para el aula convencional y 0,9144 para el aula modificada. Todo esto demuestra que los sujetos de estudio (alumnos) perciben la sensación térmica de acuerdo a la temperatura del ambiente. En la Tabla 2 se muestra los valores calculados

Tabla 2

Valor neutral y rangos de confort térmico por temperatura, aula convencionaly modificada, I.E.S. Pacaje

\begin{tabular}{|ccc}
\hline Característica & $\begin{array}{c}\text { Aula convencional } \\
\left({ }^{\circ} \mathrm{C}\right)\end{array}$ & $\begin{array}{c}\text { Aula modificada } \\
\left({ }^{\circ} \mathrm{C}\right)\end{array}$ \\
\hline TAn+2DS & 10,6 & 15,43 \\
\hline TAn+1DS & 9,6 & 14,43 \\
\hline TAn Media & 8,6 & 12,82 \\
\hline TAn-1DS & 7,6 & 11,21 \\
\hline TAn-2DS & 6,6 & 9,6 \\
\hline Rango extenso & 4.0 & 5,83 \\
\hline Rango reducido & 2.0 & 3,22 \\
$\mathrm{R}^{2}$ & 0.9264 & 0.9144 \\
\hline
\end{tabular}

TAn: Temperatura ambiente neutral, DS: Desviación estándar

$\mathrm{R}^{2}$ : Coeficiente de determinación de línea de regresión media 
Las sensaciones térmicas de frío y mucho frío se percibieron en mayor porcentaje en el aula convencional, mientras que en el aula modificada estas fueron de: ni calor ni frío con un 51,4\%. Así mismo, en el aula convencional se evidenció que los casos de calificación de sensación térmica algo de calor, calor y mucho calor no presentaron registros de valoraciones por parte de los sujetos de estudio (alumnos). Esto se debe a que las temperaturas en el mes de junio (periodo de evaluación) registran valores mínimos inclusive valores negativos por lo que la percepción de los estudiantes no mostró estos casos de valoración en el aula convencional. Lo mencionado se muestra en la Tabla 3 de contingencia cruzada.

Tabla 3

Contingencia cruzada de sensación térmica en aula convencional y modificada, I.E.S. Pacaje

\begin{tabular}{|c|c|c|c|c|c|c|c|}
\hline \multicolumn{3}{|c|}{$\begin{array}{l}\text { algo de frío } \\
\text { ni calor ni frío }\end{array}$} & $\begin{array}{c}\text { algo de } \\
\text { calor }\end{array}$ & Aula mo & ícada & & Total \\
\hline \multirow{8}{*}{ 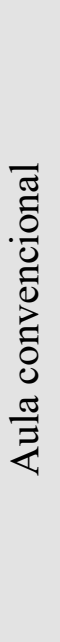 } & \multirow{2}{*}{$\begin{array}{l}\text { mucho } \\
\text { frío }\end{array}$} & Recuento & 1 & 7 & 2 & 3 & 13 \\
\hline & & $\begin{array}{c}\% \text { del } \\
\text { total }\end{array}$ & $2,7 \%$ & $18,9 \%$ & $5,4 \%$ & $8,1 \%$ & $35,1 \%$ \\
\hline & \multirow[t]{2}{*}{ frío } & Recuento & 2 & 8 & 2 & 2 & 14 \\
\hline & & $\begin{array}{c}\% \text { del } \\
\text { total }\end{array}$ & $5,4 \%$ & $21,6 \%$ & $5,4 \%$ & $5,4 \%$ & $37,8 \%$ \\
\hline & \multirow{2}{*}{$\begin{array}{l}\text { algo de } \\
\text { frío }\end{array}$} & Recuento & 1 & 4 & 3 & 0 & 8 \\
\hline & & $\begin{array}{c}\% \text { del } \\
\text { total }\end{array}$ & $2,7 \%$ & $10,8 \%$ & $8,1 \%$ & $0,0 \%$ & $21,6 \%$ \\
\hline & \multirow{2}{*}{$\begin{array}{l}\text { ni calor } \\
\text { ni frío }\end{array}$} & Recuento & 0 & 0 & 1 & 1 & 2 \\
\hline & & $\begin{array}{c}\% \text { del } \\
\text { total }\end{array}$ & $0,0 \%$ & $0,0 \%$ & $2,7 \%$ & $2,7 \%$ & $5,4 \%$ \\
\hline \multirow{2}{*}{\multicolumn{2}{|c|}{$\begin{array}{c}\text { Total } \\
\% \text { del total }\end{array}$}} & Recuento & 4 & 19 & 8 & 6 & 37 \\
\hline & & $10,8 \%$ & $51,4 \%$ & $21,6 \%$ & $16,2 \%$ & $100,0 \%$ & \\
\hline
\end{tabular}

La sensación térmica percibida en el aula convencional mostró casos fuera de los límites hacia el extremo del rango extenso mayor ( $+2 \mathrm{DS})$, lo que indica menor grado de adaptación a temperaturas frías que coincidió con lo expresado por (Fanger, 1973; ISO 7730, 2005). Así mismo, se evidenció que la sensación térmica va reduciéndose con la valoración a frío. Esto incrementa la desviación estándar con unaaproximación importante de la recta de regresión en función a la recta de regresión media que demuestra una menor adaptación a las condiciones ambientales frías por parte de los alumnos evaluados. Lo descrito es similar a lo señalado por los autores Nikolopoulou y Steemers (2003), Así, también, se presentó una reducción en la temperatura, de mucho frío con respecto a, ni calor ni frío, lo cual se debe a las condiciones de la envolvente infraestructura existente. Esta situación es similar a lo señalado por Mondelo et al., 2001 y Fanger, 1973. Lo citado se observa detalladamente en la Figura 3 y Tabla 4. 


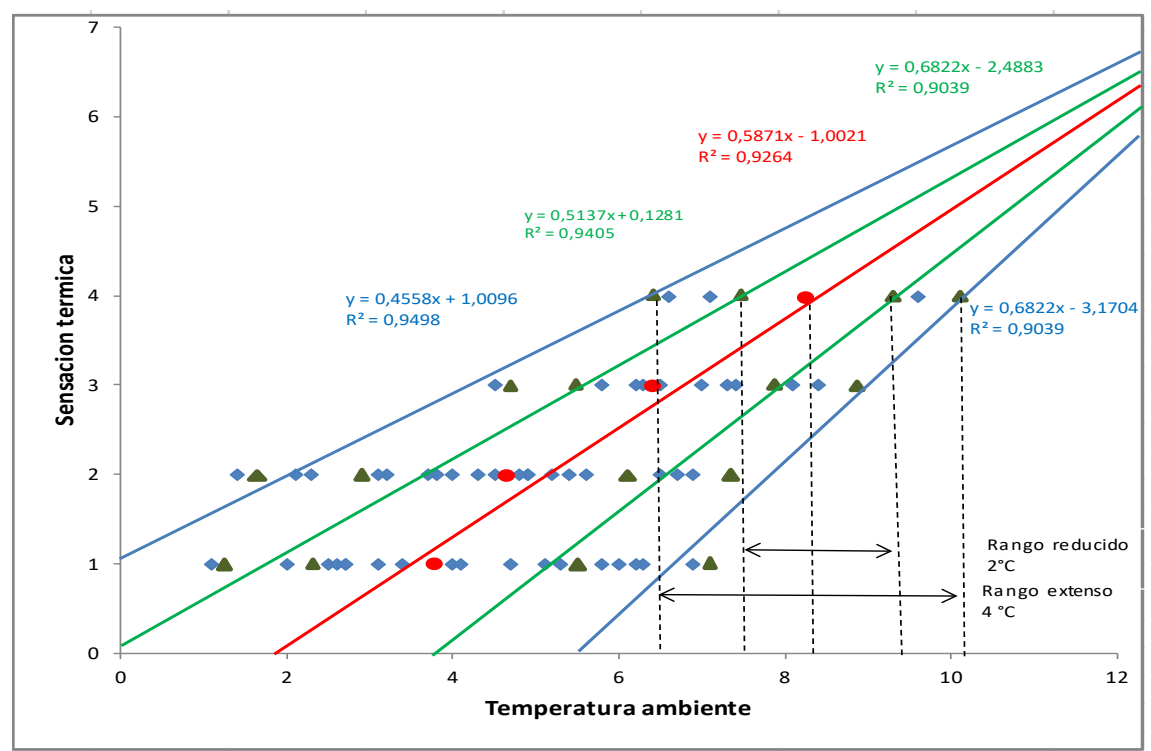

Figura 3. Línea de regresión de sensación térmica y temperatura en aula convencional, I.E.S. Pacaje

Tabla 4

Temperatura neutral media y rangos de confort, aula convencional, I.E.S. Pacaje

\begin{tabular}{cccccccc}
\hline DS & $\begin{array}{c}\text { Sensación } \\
\text { térmica }\end{array}$ & Escala & -2DS & -1DS & Media & +1DS & ++2DS \\
\hline 1 & Ni calor ni frío & 4 & 6,6 & 7,6 & 8,6 & 9,6 & 10,6 \\
1,15 & Algo de frío & 3 & 4,45 & 5,6 & 6,75 & 7,9 & 8,9 \\
1,52 & Frío & 2 & 1,34 & 2,86 & 4,38 & 5,9 & 6,9 \\
1,72 & Mucho frío & 1 & 0,69 & 2,41 & 4,13 & 5,85 & 6,85 \\
\hline
\end{tabular}

Por otro lado, se evidenció en el aula convencional una tendencia de convergencia de las líneas de regresión hacia la línea de regresión media conforme aumentó la sensación térmica percibida de frío a ni calor ni frío, lo que indica una mejor adaptación a estas condiciones de temperatura. Esto fue similar a lo mencionado por diferentes autores como (Humphreys y Nicol, 2000 y Nikolopoulou y Steemers, 2003).

El aula modificada con tecnologías bioclimáticas no presentó valoraciones para el rango de mucho calor. Así mismo, no se registraron valoraciones para la sensación térmica de frío y mucho frío, esto debido a que los sujetos de estudio percibieron la mejora de la temperatura del aula tras el tratamiento (Figura 3). Por otro lado, se evidenció que las líneas de regresión fueron ligeramente convergentes con respecto a línea de regresión media conforme se incrementó la sensación térmica a calor lo cual permite afirmar que existe una adaptación a estas condiciones y coincide con lo seńalado por Nikolopoulou y Steemers, 2003.

Del mismo modo, en el aula modificada, la sensación térmica de ni calor ni frío presentó una dispersión considerable con relación a otros rangos de sensaciones térmicas por lo que se infiere un menor nivel de adaptación de los sujetos estudiados. 
Esto es corroborado por Fanger, 1973 y Humphreys et al., 2007. De igual manera, los rangos extensos y reducidos mostraron una variabilidad significativa en las condiciones de adaptación térmica de los sujetos de estudio concordante con lo señalado por Humphreys y Nicol, 2000 quienes mencionan que los sujetos en un ambiente determinado están influenciados por otros aspectos como psicológicos y fisiológicos.

Las líneas de regresión fueron convergentes ligeramente hacia el rango de calor con respecto a la línea de regresión media, lo que indica condiciones similares de percepción de los sujetos con respecto al ambiente térmico tanto para condiciones de calor, algo de calor, ni calor ni frío. Así mismo, en las escalas de sensación térmica, se observó una similitud entre los valores centrales (algo de calor, ni calor, ni frío) que demuestra una tendencia hacia el confort térmico de los sujetos en estas condiciones. Estos resultados se exponen en la Figura 4 y Tabla 5.

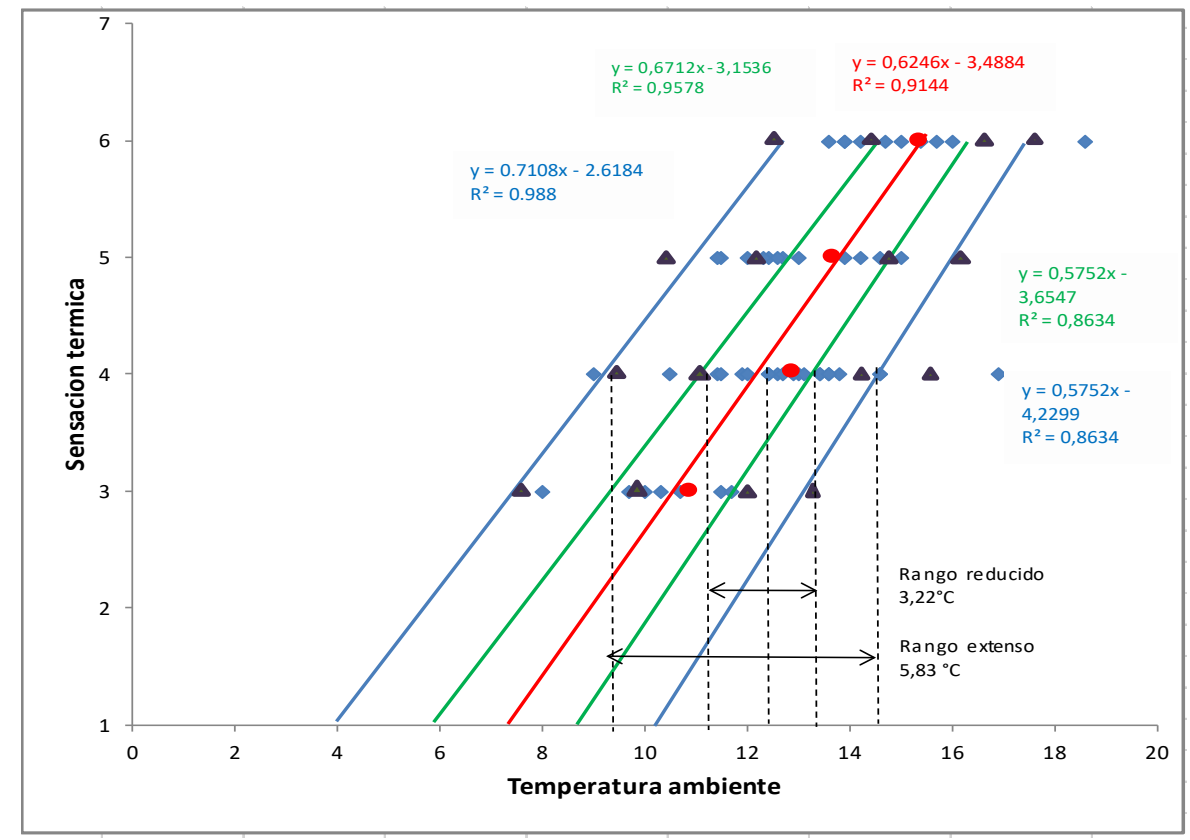

Figura 4. Línea de regresión de sensación térmica y temperatura aula modificada, I.E.S. Pacaje

Tabla 5

Temperatura neutral media y rangos de confort, aula modificada, I.E.S. Pacaje

\begin{tabular}{cccccccc}
\hline DS & $\begin{array}{c}\text { Sensación } \\
\text { térmica }\end{array}$ & Escala & $\mathbf{- 2 D S}$ & $\mathbf{- 1 D S}$ & Media & +1DS & .+2DS \\
\hline 1,5 & Calor & 6 & 12,1 & 13,6 & 15,1 & 16,6 & 17,6 \\
1,2 & Algo de calor & 5 & 10,57 & 11,77 & 12,97 & 14,17 & 15,17 \\
1,61 & Ni calor ni frío & 4 & 9,6 & 11,21 & 12,82 & 14,43 & 15,43 \\
1,24 & Algo de frío & 3 & 7,79 & 9,03 & 10,27 & 11,51 & 12,51 \\
\hline
\end{tabular}


Analizando comparativamente las variables de estudio, se observó una mayor diferencia entre los valores de temperatura neutral del aula convencional y el aula modificada que se deben a la incorporación de tecnologías bioclimáticas y al cerramiento de la envolvente del aula convencional donde se presenta una mayor adaptación entre los sujetos evaluados. Mientras que se observa menor adaptación de los sujetos estudiados en el aula convencional. Esta situación es lógica en base a su conducta reactiva, experiencia y expectativa. Lo anteriormente descrito coincide con los estudios de (Humphreys y Nicol,
2000; Nikolopoulou y Steemers, 2003 y Mondelo et al., 2001).

Así mismo, la línea de regresión media nos indica que existe una relación muy alta entre la temperatura y la sensación térmica percibida por parte del sujeto estudiado (alumnos). Del mismo modo, la pendiente de la línea de regresión es positiva, lo que revela que la relación de la sensación térmica y la temperatura son directamente proporcionales por lo que se infiere que entre más aumente la variable sensación térmica, más se incrementa la variable temperatura ( Tabla 6).

Tabla 6

Linea de regresión media de aula convencional y modificada, I.E.S. Pacaje

\begin{tabular}{cccc}
\hline Variable meteorológica & $\begin{array}{c}\text { Características de } \\
\text { la línea de } \\
\text { regresión media }\end{array}$ & $\begin{array}{c}\text { Aula } \\
\text { convencional }\end{array}$ & $\begin{array}{c}\text { Aula } \\
\text { modificada }\end{array}$ \\
\hline Pendiente de la recta & 0,5871 & 0,6446 \\
Temperatura ambiente & $\begin{array}{c}\text { Término } \\
\text { independiente } \\
\mathbf{R}^{\mathbf{2}}\end{array}$ & $-1,0021$ & $-5,4884$ \\
\hline
\end{tabular}

$\mathrm{R}^{2}$ : Coeficiente de determinación de la línea de regresión media

La sensación térmica de confort por temperatura de ambiente puede variar, depende del nivel de actividad. Una actividad intensa presenta una variabilidad alta. Para este estudio, la actividad que se consideró fue pasiva, lo que indica que la sensación térmica percibida no presenta mucha variabilidad, esto de acuerdo a Humphreys y Nicol, 2000.

\section{Conclusiones}

De acuerdo a los resultados, se puede afirmar que el confort térmico está directamente relacionado con la sensación de bienestar del individuo en un ambiente térmico.

La sensación térmica percibida presentó diferencias significativas, la temperatura media neutral calculada fue de $8,6^{\circ} \mathrm{C}$ para el aula convencional y para el aula modificada fue de $12,82^{\circ} \mathrm{C}$. Esto revela la existencia de una mejora en la percepción por parte de los sujetos estudiados.

La regresión lineal calculada fue 0.9264 para el aula convencional y 0.9144 para el aula modificada. Estos valores evidenciaron una relación muy alta entre la temperatura y la percepción del sujeto. 


\section{Referencias}

Bojórquez, G. (2010). Confort térmico en exteriores: Actividad en espacios recreativos en clima cálido seco extremo. Universidad de Colima.

Basso, M.; Fernández, J. y De Rosa, C. (2000). Evaluación térmica y económica comparativa de crujias de aulas en edificios escolares bioclimáticos en la provincia de Mendoza. Laboratorio de Ambiente Humano y Vivienda - Instituto Ciencias Humanas Sociales y Ambientales.

Brager, G. and De Dear, R. (1998). Thermal adaptation in the buil environment. A literature review. Energy and Buildings, 27, 83-96.

Del Campo, V. y Mendivil, A. (2003). Calidad del aire interior en los centros de educación infantil del país Vasco. Universidad del País Vasco. ETS de Ingenieros, Alda. Urquijo 48013 Bilbao.

Fanger, O. (1973). Thermal Comfort. Analysis and aplicattions in environmental engineering, Ed. McGraw - Hill.

Gómez-Azpeitia, G.; Ruiz, R.; Bojórquez, G. y Romero, R. (2007). Monitoreo de condiciones de confort térmico. Comisión Nacional del Fondo para Vivienda. Proyecto: Confort térmico y ahorro de energía en la vivienda económica - México. CONAFOVI. 200401-20). Colima, Colima.
Humphreys, M. and Nicol, F. (1998). Understanding the adaptive approach to thermal comfort. ASHRAE Transactions, Technical Bulletin, 104 (1) pp 991- 1004.

Humphreys, M. and Nicol, F. (2000). Outdoor temperature and indoor thermal comfort. Raising the precision of the relationship for the 1998. ASHRAE. Database of field studies. ASHRAE. Transactions 206, (2), 485 - 492.

Humpreys, M.; Nicol, F. and Raja, I. (2007). Field studies of indoor thermal comfort and the progress of the adaptative approach. Advances in building energyresearch. Earthscan.

ISO 10551, (1995). International Organization for Standarization. Ergonomics of thermal enviroment - assessment of the influence of the thermal environment using subjective judgement scales.

ISO 7730, (2005). International Organization for Standarization. Ergonomics of the thermal environment-analytical: Determination and interpretation of thermal comfort, using calculation of the PMV and PPD indices and local thermal. Ginebra.

Ledesma, S. L.; Viviana, M.; Cisterna, M. y Cecilia F (2015). Habitabilidad en aulas escolares en Tafí del Valle, Tucumán. Propuestas para mejorar su acondicionamiento 
térmico. Energías Renovables $y$ Medio Ambiente. Vol. 3.

Mercat, M.; Ledesma, S. y Gonzalo, G. (2016). Centro de Estudios Energía, Hábitat y Arquitectura Sustentable. Instituto de Acondicionamiento Ambiental. Facultad de Arquitectura y Urbanismo - Universidad Nacional de Tucumán Avances en Energías Renovables y Medio Ambiente Vol. 20, pp 08.4708.59 .

Maxwell, L. E. (2016). School building condition, social climate, student attendance and academic achievement: A mediation model. Journal of Environmental Psychology. Vol.46, June 2016, Pages 206-216. Elsevier.

Miranda, L. G. (2008). Estudio térmico del Edificio de Ingeniería Civil de la Universidad de Chile. Ed. Universidad de Chile.

Móndelo, P.; Gregori, E.; Comas, S.; Castejón, E. y Bartolomé, E. (2001). Ergonomia 2: Confort y estrés térmico. 3ra. Edición. Universitat Politécnica Catalunya.

Nicol, F. and Humphreys, M. (1998). Adaptive thermal comfort and sustainable thermal standars for buildings. Energy and Buildings, 34. 563-572.

Nikolopoulou, M. (2004). Designingopen space in the urban environment: a bioclimatic approach. Attiki: Center for renewable energy sources.
Nikolopoulou, M. and Steemers, K. (2003). Thermal comfort and psychological adaptation as a guide for designing urban spaces. Energy and Buildings, 35, 95-101.

Pellegrino, A. (2015). Daylighting for Green Schools: A Resource for Indoor Quality and Energy Efficiency in Educational Environments. Energy Procedia. Vol. 78, November 2015, Pages 3162- 3167. 6th International Building Physics Conference.

Pulgar, J. (1987). Geografía del Perú. Las Ocho Regiones Naturales. 9 ed. Lima. Promoción Editorial Inca, S.A. p. 27-176.

Rincón, J. M. y Bojórquez, G. M. (2014). Estimación del confort térmico a partir del enfoque adaptativo: estudio en sitio. Congreso XXXVIII Semana Nacional ANES - XI Congreso Iberoamericano.

Ruiz-Torres, P. (2007). Estándar de confort térmico para la ciudad de Colima. [Tesis de Maestría]. Universidad de Colima.

Shamsuddin, S. (2012). Relationship between the Outdoor, Physical Environment and Students' Social Behaviour in Urban Secondary School. Procedia-Social and Behavioral Sciences. Vol.50, 2012, Pages 148-160.

Tsuzuki, K. y Ohfuku, T. (2002). Thermal sensation and thermoregulation, in el derly compared to young people in Japanese winter season. National Institute of Advanced Industrial 
Science and Technology, Tsukuba, JAPAN.

Walter, E.; Agüero, M.; Watkins, M. y Mansilla, G. (2011). Escuela Bioclimática y Sustentable SECUNDARIA No 39 - Villa de Antofagasta de la Sierra Catamarca. Acta del I Encuentro
Nacional sobre Ciudad, Arquitectura y Construcción Sustentable.

Wargocki, P. y Wyon, D. P. (2006). Research report on effects of HVAC on student performance. ASHRAE Journal 48: p. 22-28. 